\title{
Empirical prediction of flap tip noise
}

\author{
Karl-Stéphane Rossignol* \\ German Aerospace Center (DLR), Lilienthalplatz 7, D-38108 Braunschweig
}

\begin{abstract}
In this paper, DLR's empirical prediction model for flap tip noise is presented and discussed in details. The prediction scheme is based on a comprehensive acoustic and aerodynamic database acquired in the Acoustic Wind Tunnel Braunschweig. It was verified, through successful scaling of the measured noise spectra, that the cross-flow velocity at the flap tip is an important parameter characterizing the flow mechanism(s) responsible for the noise production. This finding led to the definition of a universal flap tip noise spectral shape in terms of a linear least-squares fit of the corresponding measurement data. Using a similar approach, a model for the flap tip noise directivity was formulated. The prediction model was compared against full-scale fly-over measurement data (B747-400 and A319) and an acceptable agreement of the overall predictions was found. A slight underprediction of the noise levels at high frequencies suggests that additional airframe noise sources might be needed in the complete aircraft noise prediction scheme to get a better agreement between measured and predicted noise levels. It is also found that, for large flap deflection angles, flap tip noise dominates the high frequency part of the predicted complete aircraft high-lift noise spectra. Knowledge of the flap tip noise peak frequency and high-frequency decay are therefore sufficient to account for this noise source in the total aircraft noise prediction. Finally, the limitations of the prediction scheme are discussed and research needs are identified.
\end{abstract}

\section{Nomenclature}

$U_{0} \quad$ Free stream velocity $[\mathrm{m} / \mathrm{s}]$

$U_{c} \quad$ Cross-flow velocity $[\mathrm{m} / \mathrm{s}]$

$\mathcal{M} \quad$ Mach number $\left(=U_{0} / a\right)$

Re Reynolds number $\left(=U_{0} c / \nu\right)$

$\mathcal{S} t_{0} \quad$ Strouhal number based on flap chord and free-stream velocity $\left(=f c / U_{0}\right)$

$S t_{c} \quad$ Strouhal number based on flap chord and cross-flow velocity $\left(=f c / U_{c}\right)$

a Speed of sound $[\mathrm{m} / \mathrm{s}]$

c $\quad$ Flap chord length $[\mathrm{m}]$

f $1 / 3$-octave band central frequency $[\mathrm{Hz}]$

$p \quad$ Acoustic pressure $[\mathrm{Pa}]$

D Directivity function $[\mathrm{dB}]$

$\delta_{f} \quad$ Flap geometrical deflection angle $\left[^{\circ}\right]$

$\nu \quad$ Kinematic viscosity $\left[\mathrm{m}^{2} / \mathrm{s}\right]$

$\phi \quad$ Polar angle $\left[{ }^{\circ}\right]$

$\rho \quad$ Density of air $\left[\mathrm{kg} / \mathrm{m}^{3}\right]$

$\theta \quad$ Azimuthal angle $\left[^{\circ}\right]$

SPL 1/3-octave band sound pressure level [dB]

$\mathrm{SPL}_{n} \quad 1 / 3$-octave band scaled sound pressure level [dB]

\section{Introduction}

$\mathrm{T}^{\mathrm{T}}$ is commonly known that an important part of the airframe noise generated by an aircraft is due to the 1 deployment of the components of its high-lift system. The flap was, already in the eighties, identified

\footnotetext{
${ }^{*}$ Research Engineer, Department of Technical Acoustics, Institute of Aerodynamics and Flow Technology, German Aerospace Center (DLR), karl-stephane.rossignol@dlr.de
} 
as a major noise contributor in the approach and landing phases. By theoretical considerations, Howe ${ }^{1}$ evaluated that noise generated at a single flap tip could be as much as $3 \mathrm{~dB}$ higher than trailing-edge (TE) noise integrated along the whole of the flap span.

Since then, many experimental ${ }^{2-8}$ and theoretical ${ }^{1,9-12}$ work on flap tip noise was done, providing a large amount of knowledge regarding its production mechanisms as well as noise reduction approaches. In regard to the current need for accurate and efficient semi-empirical airframe noise prediction tools, only a few authors ${ }^{3,6}$ have proposed appropriate models for flap tip noise. This scarcity may be explained by the non-triviality to assess it in wind tunnels. For open-section wind tunnels, the effect of the shearlayers on noise propagation is a major problem to be overcome. The sole presence of the shear-layers also limits greatly the spatial extent where measurements can effectively be performed. Moreover and especially for multi-element high-lift system models, the occurence of many spurious noise sources as well as the presence of other loud components renders the isolation of flap tip noise very difficult. In closed section wind tunnels, low signal to noise ratio (SNR) due to dominant wall boundary layer hydrodynamic pressure fluctuations are also major causes of concern.

In a previous work done by the author, ${ }^{13}$ the aerodynamic and acoustic characteristics of a single flap tip (see figure 1) were studied. The acoustic measurements have revealed the most important spectral characteristics of the model tip noise source while providing a preliminary description of its directivity. Based upon these findings an empirical prediction model for noise produced by an isolated flap tip was formulated and implemented. In this paper a description of the prediction scheme is given along with details regarding its limitations and the assumptions made in the process.

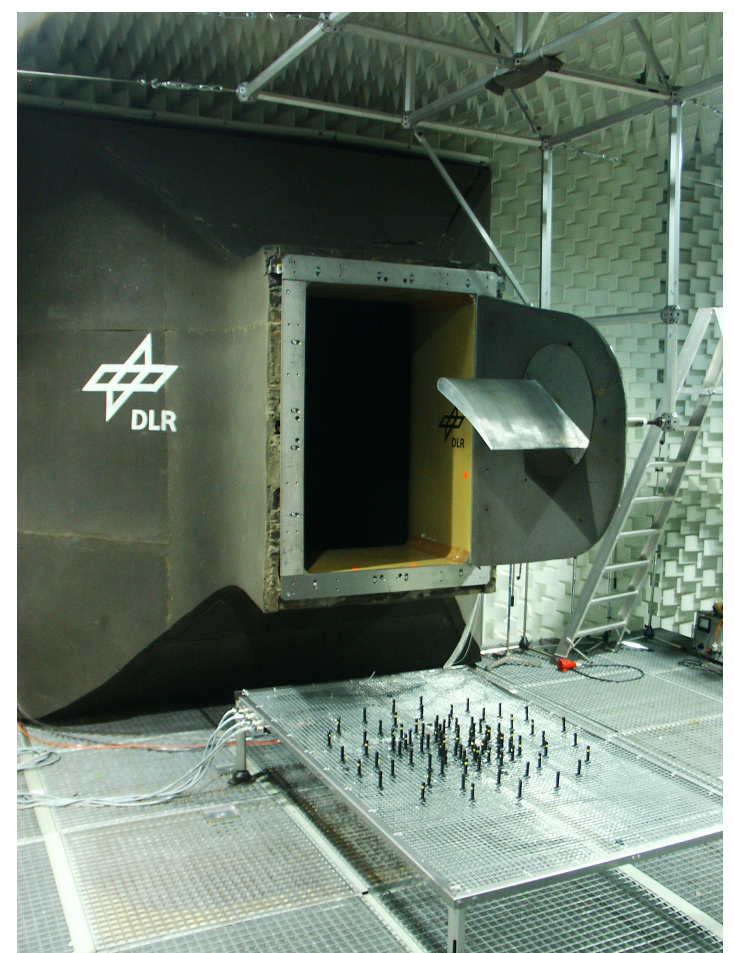

(a) Large aperture array (96 1/2" LinearX M51 microphones).

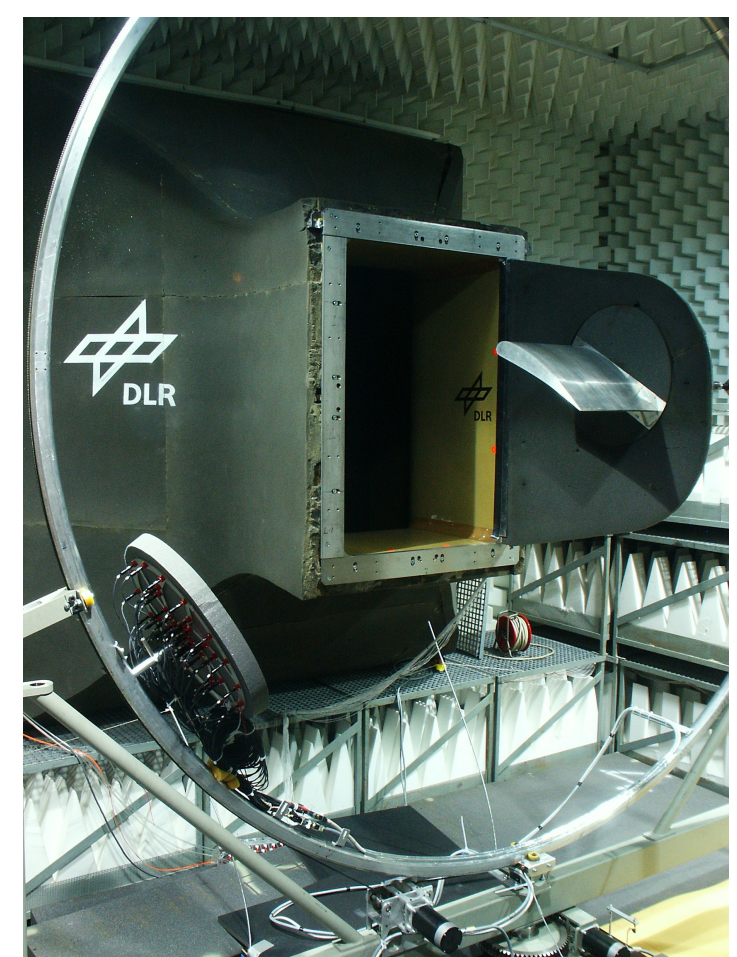

(b) Small aperture array on its positioning system (48 1/4" LinearX M31 microphones).

Figure 1: Isolated flap model (modified A320 flap geometry). Experimental setups for the large and small aperture microphone arrays.

\section{Experimental measurements}

Measurements performed in 2008 (see Rossignol ${ }^{13}$ for more details regarding the experiment) and 2011 in DLR's anechoic wind tunnel in Braunschweig, Germany (AWB) consitute the database used for the subsequent development of DLR's flap tip noise prediction model. The AWB is an anechoic open-jet wind tunnel capable of running at speeds of up to $U_{0}=65 \mathrm{~m} / \mathrm{s}$. It is optimized for noise measurements at frequencies above $250 \mathrm{~Hz}$.

The prediction model described in the following sections is based solely on acoustic and aerodynamic measurements made at an isolated flap having a modified A320 geometry. This model is nearly in full 
scale dimensions with a flat square tip geometry which ensures the formation of a well developed sideedge vortex system and a correspondingly loud noise source which can be easily quantified using the microphone array technique. The model was mounted as a cantilever wing in the open test section of the wind tunnel using only one supporting side-plate (see figure 1).

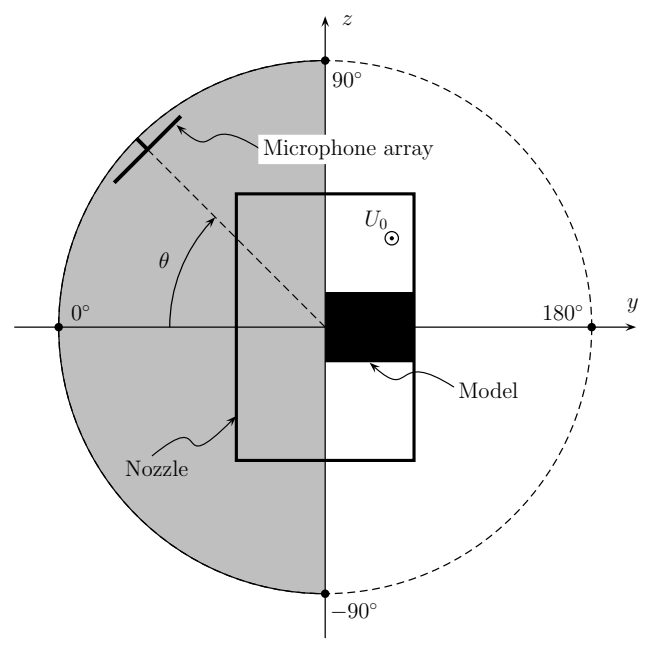

(a) Looking upstream at the model.

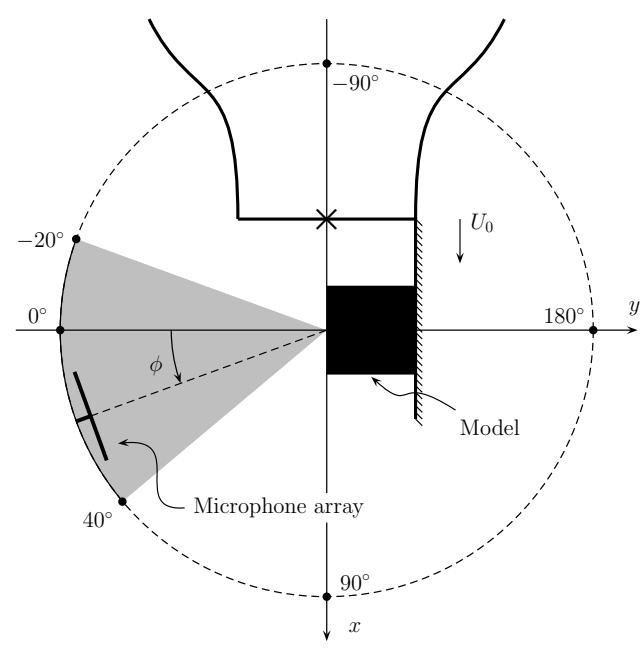

(b) Looking down at the model.

Figure 2: Spatial extent of the directivity measurements (from Rossignol ${ }^{13}$ ).

Sound source localization as well as quantification was performed using both large and small aperture microphone arrays (see Rossignol ${ }^{13}$ ). Measurements were done for Mach numbers ranging from $\mathcal{M}=$ 0.087 to 0.175 , corresponding to Reynolds numbers (based on the flap chord) ranging from $\mathcal{R} e=0.96 \times 10^{6}$ to $1.92 \times 10^{6}$ (see table 1 ).

Boundary layer tripping was used on the suction side of the isolated flap at $x / c=0.01$ and on its pressure side at $x / c=0.34$ to reduce the appearance of TE tones as well as to ensure the development of fully turbulent boundary layers and prevent premature separation of the boundary layers. Boundary layer separation was first observed for $\delta_{f}=30^{\circ}$. For this configuration a small zone of detached flow exists near the supporting plate at the TE and on the suction side of the model.

\begin{tabular}{ccc}
$U_{0} \mathrm{~m} / \mathrm{s}$ & $\mathcal{M}$ & $\mathcal{R} e$ \\
\hline 40 & 0.117 & $1.28 \times 10^{6}$ \\
50 & 0.146 & $1.60 \times 10^{6}$ \\
60 & 0.175 & $1.92 \times 10^{6}$ \\
\hline
\end{tabular}

Table 1: Non-dimensional flow parameters. $a=343 \mathrm{~m} / \mathrm{s}, \rho=1.204 \mathrm{~kg} / \mathrm{m}^{3}, \nu=1.5 \times 10^{-5} \mathrm{~m}^{2} / \mathrm{s}$.

During the 2008 measurement campaign ${ }^{13}$ information regarding the mean flow field in the vicinity of the model tip was gathered at numerous streamwise position using a 7-hole probe. Based on these measurements the dependendcy of the cross-flow velocity $\left(U_{c}\right)$ on the model flap angle was established. $U_{c}$ is the local spanwise mean flow velocity at the flap tip lower edge and a chordwise position of $x / c=0.7$. The maximum value of $U_{c}$ being reached at that chordwise position, nearly independent of $\delta_{f}$. This parameter was shown to be representative of the flow mechanism leading to noise radiation at the flap tip. $^{13}$

\section{Data acquisition and processing}

Two different microphone arrays were used for the acoustic measurements: a small aperture array consisting of 48 1/4" LinearX M31 microphones and a large aperture array having 96 1/2" LinearX M51 microphones. Both experimental setups are shown in figure 1. The first array is made of a metal plate covered with a five centimeter thick foam sheet in order to reduce sound reflections. The transducers are flush mounted through the foam. For the second array a wire grid is used to support the microphones. A computer controlled traversing system enables a precise positioning of the small aperture array around the model (see figure 1). The range of the measurements is summarized in table 2, whereas measurement 
coordinates are defined in figure 2. The given ranges correspond to the maximal displacements attainable considering the physical constraints imposed by the wind tunnel nozzle. For the second array a wire grid is used to support the microphones.

Data acquisition was done using two 48-channel GMB Viper measuring units at a sampling rate of $45.0 \mathrm{kHz}$. Measurement duration was set to 22 seconds, which gives a total of 245 blocks of 4096 samples, for a frequency resolution of $10.99 \mathrm{~Hz}$.

\begin{tabular}{cccccccc} 
& \multicolumn{3}{c}{$\theta\left[^{\circ}\right]$} & & \multicolumn{3}{c}{$\phi\left[^{\circ}\right]$} \\
\hline & $\min$ & $\max$ & $\Delta$ & & $\min$ & $\max$ & $\Delta$ \\
\cline { 2 - 4 } \cline { 6 - 8 } $48 \mathrm{M}$ & -90 & 90 & 15 & & -20 & 40 & 10 \\
$96 \mathrm{M}$ & -90 & 0 & 90 & & 0 & 0 & 0 \\
\hline
\end{tabular}

Table 2: Range of the directivity measurements for the isolated flap model.

Noise source identification was done using conventional beamforming ${ }^{14}$ (CB). To quantify the noise level a of particular source, power integration as described by Brooks et al. ${ }^{15}$ was used. For the present work, noise levels lower than $10 \mathrm{~dB}$ below the peak sound pressure level (SPL) value were excluded from the integration. The scanning grid (and integration region) was chosen to be parallel to the $x-z$ plane or to the $x-y$ plane depending on microphone array location. Also, extensive use of diagonal removal (DR) is made to reduce the noise floor level induced by the microphones auto-powers.

Background noise correction was performed down to $3 \mathrm{~dB}$ differences. The background noise is taken here as the noise measured at $\delta_{f}=0^{\circ}$. Noise spectra were also (prior to background noise subtraction) corrected for convective amplification, source convection and shear-layer diffraction. Hereby, use of the well-known shear-layer correction developed by Amiet ${ }^{16}$ was made. Finally, sound pressure levels were back-propagated to a reference distance of $1 \mathrm{~m}$ from the model.

\section{Flap tip noise spectral shape}

Based on the present measurements and also from previous measurements reported in Rossignol ${ }^{13}$ an empirical prediction model for flap tip noise was developed. Its formulation accounts for the influence of both free-stream velocity $\left(U_{0}\right)$ as well as flap deflection angle $\left(\delta_{f}\right)$ and also includes noise source directivity effects.

Noise intensity is assumed to follow a power law of the form $p^{2} \propto U_{c}^{n}$ when plotted versus a Strouhal number $\left(\mathcal{S} t_{c}=f \cdot c / U_{c}\right)$ based on flap chord $(c)$ and the tip cross-flow velocity $\left(U_{c}\right)$ as the relevant local velocity. $U_{c}$ is taken here as the spanwise component of velocity at the flap tip lower ridge level ${ }^{13}$ and $x / c=0.7$. It is obtained from the following empirical relation:

$$
\frac{U_{c}}{U_{0}}=\frac{0.0316 \cdot \delta_{f}}{\delta_{r e f}}+0.1879
$$

$\delta_{\text {ref }}$ is a reference deflection angle $\left(\delta_{\text {ref }}=1^{\circ}\right)$. For noise spectral scaling, the cross-flow velocity is non-dimensionalized by an arbitrary reference velocity of $U_{r e f}=100 \mathrm{~m} / \mathrm{s}$. The best collapse of the data is obtained for an exponent of $n=5.5$ (see figure 3). From the preceding arguments a normalized flap tip noise spectra can be expressed as follows;

$$
\mathrm{SPL}_{n}=\mathrm{SPL}+20 \cdot \log _{10}(r / c)-55 \cdot \log _{10}\left(U_{c} / U_{\text {ref }}\right)
$$

From the theory of aeroacoustic noise the generation of noise through interaction of turbulence with an infinitely thin edge should result in a velocity dependance to an exponent of 5.0. Higher exponent were, however, also found in other experimental investigations. ${ }^{4,7}$

The first term on the right-hand side of equation 2 accounts for the geometrical extent $(c)$ of the model and its distance from an observer $(r)$. It is assumed that noise intensity varies linearly with the radiating surface $\left(p^{2} \propto c^{2}\right)$. This assumption is based on the idea that $p^{2}$ should be proportional to the length of the flap tip and to a characteristic length of the noise-generating flow mechanism. The vortex size, which is proportional to the model chord length.

In figure 3, the scaled sound pressure levels (measured underneath the model) are plotted along with a polynomial fit. The low Strouhal number range is fitted with a $6^{\text {th }}$ order polynomial while for the high Strouhal number range a polynomial of order 3 is used (see equations 3 and 4 ). The fitted polynomials intersect at $\mathcal{S} t_{c} \approx 25$. This defines the flap tip noise spectral shape, as measured underneath the model. 


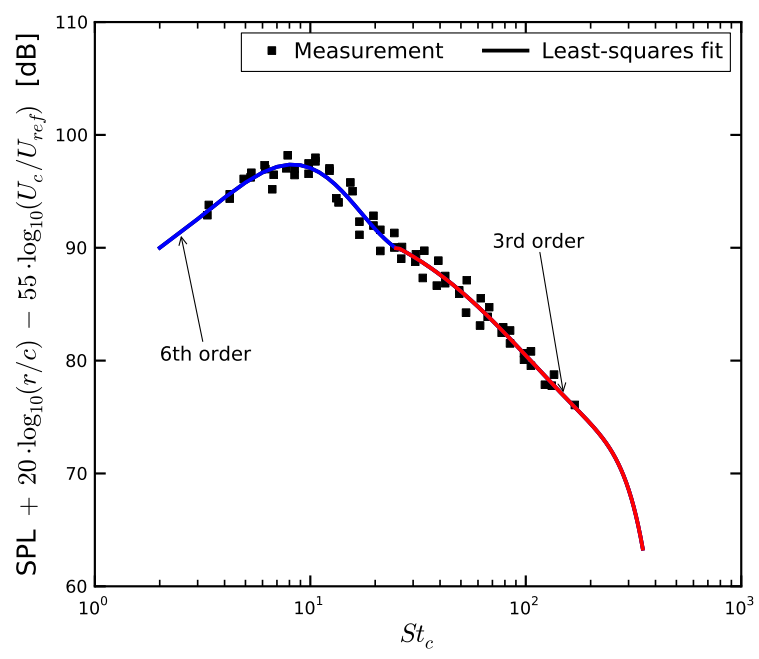

Figure 3: Scaled airfoil tip 1/3-octave band noise spectra measured underneath the model. The solid line is a least-squares fit of the data.

This approximation is independent of $\delta_{f}$ as well as $U_{0}$. A listing of the coefficients values obtained from the regression analysis is given in appendix $\mathrm{A}$.

$$
\begin{gathered}
\left.\mathrm{SPL}_{n}\left(\mathcal{S} t_{c}\right)\right|_{S t_{c} \leq 25}=l_{0}+l_{1} \cdot \mathcal{S} t_{c}+l_{2} \cdot \mathcal{S} t_{c}^{2}+l_{3} \cdot \mathcal{S} t_{c}^{3}+l_{4} \cdot \mathcal{S} t_{c}^{4}+l_{5} \cdot \mathcal{S} t_{c}^{5}+l_{6} \cdot \mathcal{S} t_{c}^{6} \\
\left.\operatorname{SPL}_{n}\left(\mathcal{S} t_{c}\right)\right|_{S t_{c}>25}=h_{0}+h_{1} \cdot \mathcal{S} t_{c}+h_{2} \cdot \mathcal{S} t_{c}^{2}+h_{3} \cdot \mathcal{S} t_{c}^{3}
\end{gathered}
$$

The spectral shape of figure 3 reveals a peak at $\mathcal{S} t_{c} \approx 9$, in good agreement with experimental data from Koop ${ }^{7}$ and Brooks et al. ${ }^{3}$ when using the same Strouhal number definition $\left(\mathcal{S} t_{c}\right)$. This, however, differs from what is found by Guo et al. ${ }^{5}\left(\mathcal{S} t_{c}=3.91\right)$. These discrepancies mightl, however, be due to a wrong evaluation of $U_{c}$ at high flap deflection angle $\left(\delta_{f}=50^{\circ} \mathrm{in}^{5}\right)$ based on equation 1 .

In figure 4 a comparison of flap tip noise spectra predictions made by three published empirical models is shown $\left(U_{0}=50 \mathrm{~m} / \mathrm{s}, \delta_{f}=25^{\circ}\right)$. In the low Strouhal number part of the spectrum all models tend towards a similar prediction. The computed spectral levels agree to about $5 \mathrm{~dB}$ with slight shift in peak frequency. Both Guo's ${ }^{6}$ and Brook's ${ }^{3}$ models predict, at high Strouhal numbers, a much more rapid SPL decay compared to the model described herein.

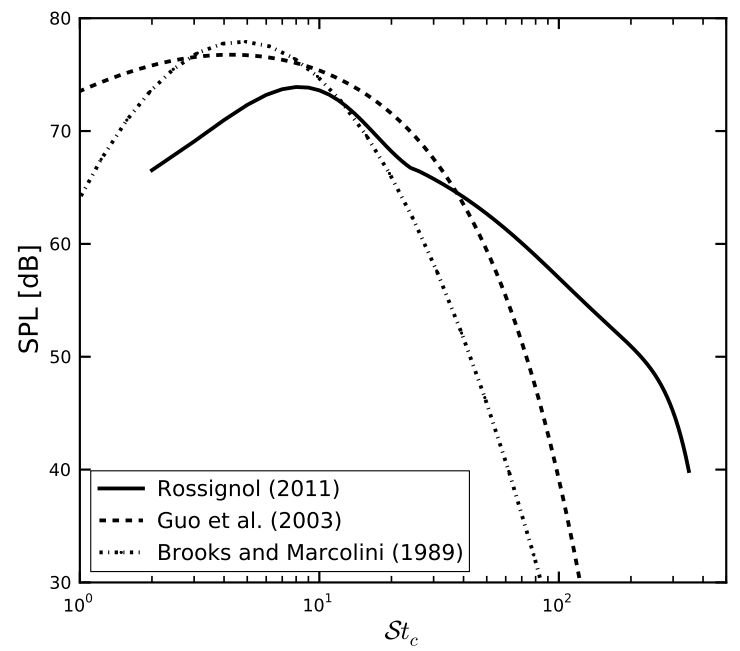

Figure 4: Comparison of existing flap tip noise prediction models. $\delta_{f}=25^{\circ}, U_{0}=50 \mathrm{~m} / \mathrm{s}$ 


\section{Flap tip noise directivity}

For the prediction of noise emitted in different directions by the flap tip, the spectral shape shown in figure 3 needs to be adjusted by a frequency, and emission angle, dependent directivity function $(D=$ $D(f, \theta, \phi)$, see figure 2 for emission angles definition). The directivity is assumed to be independent of $\delta_{f}$. This is justified by the weak dependency of the measured noise levels on this parameter. $D=D(f, \theta, \phi)$ is obtained by power integration of the small aperture microphone array data in the flap tip region. The integrated noise levels are normalized by substraction of the noise level at $\theta=-90^{\circ}, \phi=0^{\circ}$. By doing so, one directly obtains a directivity factors on (part of) a spherical surface around the model (see figure 5). D applies a level correction (in $\mathrm{dB}$ ) to the noise levels emitted by the model at $\theta=-90^{\circ}, \phi=0^{\circ}$, computed according to equations 3 and 4 .

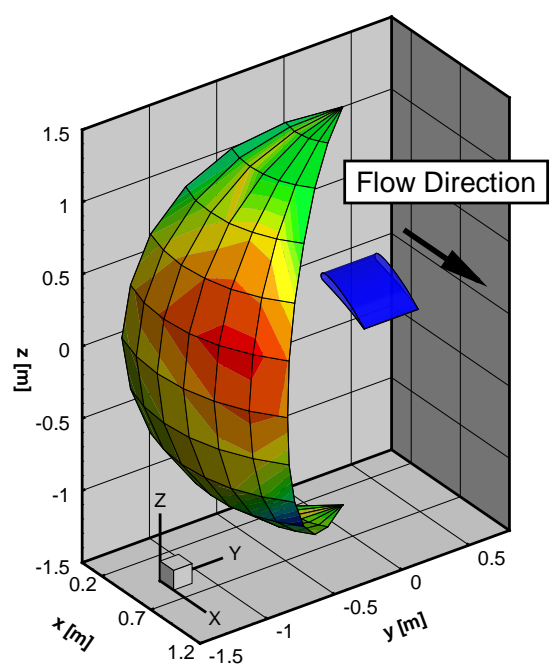

Figure 5: Directivity measurement domain.

The dependence of the directivity function on $\theta$ and $\phi$ is modeled using a two-dimensional polynomial fit of the measurements. For the present case two $3^{r d}$ order polynomials in $x$ and $y$ are used to compute the directivity factor for a given emission angle. This requires the knowledge of eight coefficients according to equation 5 .

$$
\left.\mathrm{D}(\theta, \phi)\right|_{f}=m_{0}+m_{1} \cdot \theta+m_{2} \cdot \theta^{2}+m_{3} \cdot \theta^{3}+k_{0}+k_{1} \cdot \phi+k_{2} \cdot \phi^{2}+k_{3} \cdot \phi^{3}
$$

The coefficients of equation 5 need to be computed for each frequency of interest. In the present case 13 $1 / 3$-octave band frequencies are chosen. A listing of the coefficients values obtained from the regression analysis is given in appendix $\mathrm{B}$, along with their respective $1 / 3$-octave band central frequencies.

A comparison of the empirical approximation and the measured directivity functions is shown in figure 6. Generally speaking, the results of figure 5 confirm the observation already made by Brooks ${ }^{4}$ that the emitted noise levels are higher over the model than underneath it. The principal emission direction is approximatly $\theta=0^{\circ}, \phi=0^{\circ}$, in spanwise direction. Maximum variations of $D$ of the order of $5 \mathrm{~dB}$ are observed in the measurements. These level variations strongly depend on frequency. The noise levels at $\theta=0^{\circ}, \phi=0^{\circ}$ are seen to increase with increasing frequency. The general shape of the directivity function tends to get more uniform with increasing frequency. This tendency is more noticeable for azimuthal variations of $D\left(D\left(f, \theta, \phi=0^{\circ}\right)\right.$. These results confirm obervations made by Hayes et al. ${ }^{17}$ who notes that for small wavelengths, the influence of the model tip diminishes and the directivity function should become more uniform. Lastly lets remind that the actual version of DLR's flat tip noise prediction model is not yet able to account for all details of the measured directivity functions, as is seen in figure 6 . This limitation increases in pertinence with decreasing frequency. Finally the validity range of the actual flap tip noise directivity model is limited to a domain ranging from $-90^{\circ} \leq \theta \leq 90^{\circ}$ and from $-20^{\circ} \leq \phi \leq 40^{\circ}$. The actual implementation of the prediction scheme assumes a symmetrical directivity function for $90^{\circ} \leq \theta \leq 270^{\circ}$. 


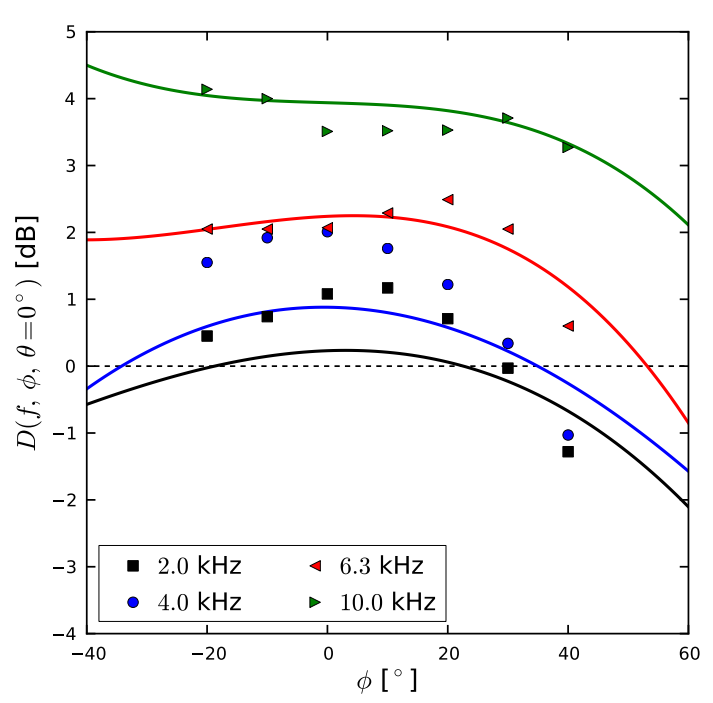

(a) Polar directivity factor.

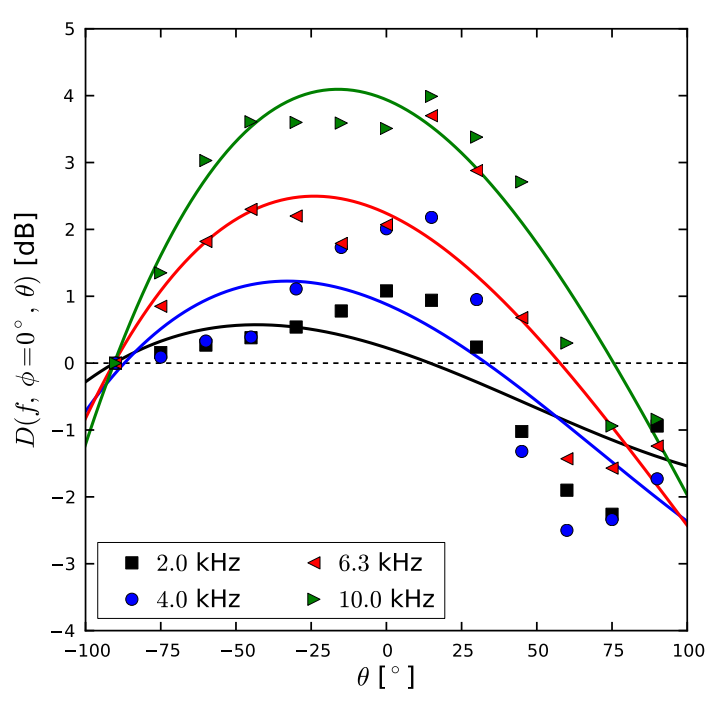

(b) Azimuthal directivity factor.

Figure 6: Comparison of measured (symbols) and empirical representation (lines) of the flap tip noise directivity function.

\section{Prediction of full-scale fly-over noise levels}

An example application of the prediction model described in the previous sections is shown in figure 7 and 8. In the figures, the solid symbols represent fly-over noise levels (overhead position, $\phi=0^{\circ}$, $\theta=-90^{\circ}$ ) of a Boeing 747-400 and A319 with slats and flaps deployed. The fly-over data include only the contributions of the clean wing (cruise configuration) plus that of the flaps and slats. Engine noise as well as landing gear noise are not accounted for in the comparison. The comparison is done for two different flap deflection angles, $20^{\circ}$ and $30^{\circ}$ in the B747-400 case and $20^{\circ}$ and $40^{\circ}$ in the A319 case. The noise levels are back-propagated to a reference distance of $1 \mathrm{~m}$. The prediction (slat and flap deployed) is shown by the dashed line. The solid line represent the contribution of the isolated flap. Note that a $20^{\circ}$ flap angle correction (increase) was applied to the prediction through equation 1 . This is justified by the fact that the result of equation 1 is based on the geometrical $\delta_{f}$ in the wind tunnel. Based on comparison between 2D (computed) and measured static pressure distributions at the model, large lift losses due to the free-stream deflection by the model are expected. The 3D character of the flow field near the flap tip is also responsible for a lift reduction in the tip vicinity. ${ }^{3}$ A more accurate estimation of the effective $\delta_{f}$ cannot be made at this stage and is left to future research efforts.

From these plots, one sees that the complete airframe noise prediction model is able to render a good estimation of the measured fly-over noise levels over most of the frequency range. Discrepencies being most important at high frequencies where noise levels are underestimated by about $5 \mathrm{~dB}$ for the B747400 and $10 \mathrm{~dB}$ for the A319. A perfect correspondance cannot, however, be awaited due to the limited number of airframe components accounted for by the complete prediction scheme. The wind tunnel measurements also indicates that two possible effects may also be responsible for the high-frequency noise underprediction shown in figure 7 and 8 . The flap tracks as well as integration effects (in the high-lift system) are aspects which are certainly important and need further investigation.

Flap tip noise is found to be responsible for the high frequency $(f>1.0 \mathrm{kHz})$ part of the spectrum while other noise sources dominates at low frequencies $(f<1.0 \mathrm{kHz})$. Knowledge of flap tip noise peak frequency and its high-frequency decay seems, therefore, to be sufficient in order to estimate the flaps contribution to the complete aircraft noise spectrum. This holds for large flap deflection angles. As shown in figure 7 and 8 , for $\delta_{f}=20^{\circ}$, the contribution of flap tip noise to the complete aircraft noise spectra is reduced and in the A319 case it becomes pratically insignificant. In this particular case, the slats are the dominating airframe noise sources.

Note that the prediction shown in figure 7 and 8 does not include the flaps which are located near the fuselage or the engines. Only free flap tips were considered. Also, small geometrical details which might be encountered on the full scale flaps and which may be important noise generators cannot be accounted for. The spectral shape defined in equation 3 and 4 is based on measurements data from a 


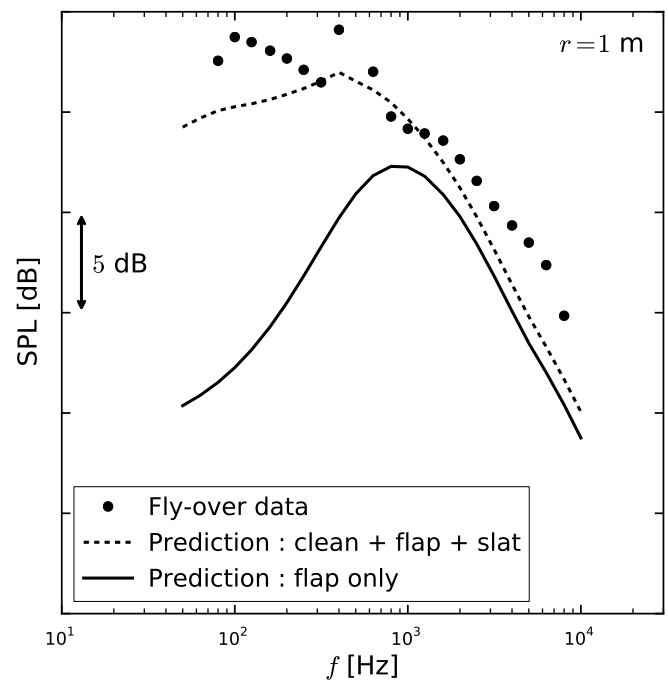

(a) $\delta_{f}=20^{\circ}, U_{0}=112.6 \mathrm{~m} / \mathrm{s}$

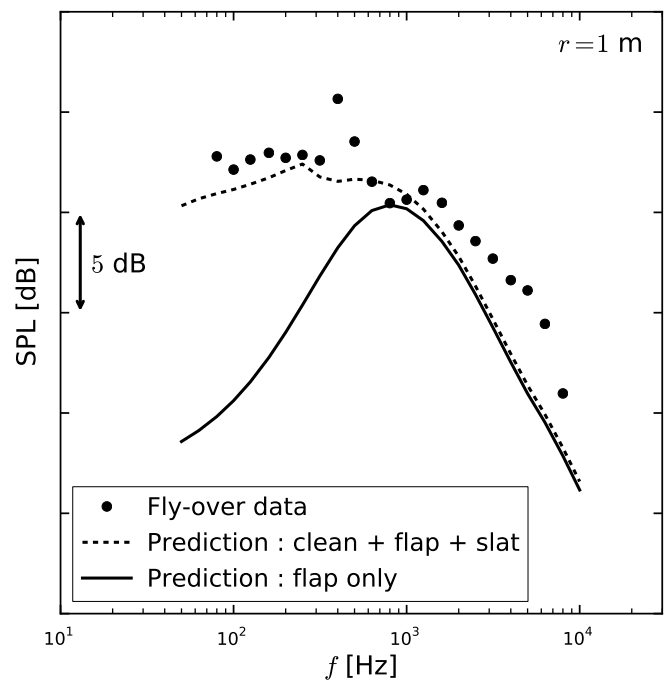

(b) $\delta_{f}=30^{\circ}, U_{0}=85.2 \mathrm{~m} / \mathrm{s}$

Figure 7: Comparison of the B747-400 fly-over noise levels (symbols) and the corresponding full-scale prediction (lines) for two flap deflection angles $\left(\delta_{f}=20^{\circ}, 30^{\circ}\right) . \phi=0^{\circ}, \theta=-90^{\circ}$.

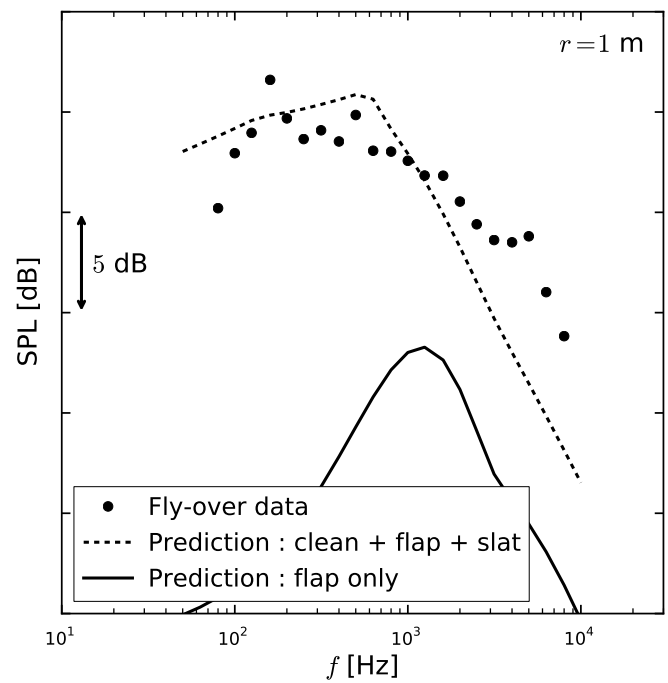

(a) $\delta_{f}=20^{\circ}, U_{0}=82.9 \mathrm{~m} / \mathrm{s}$

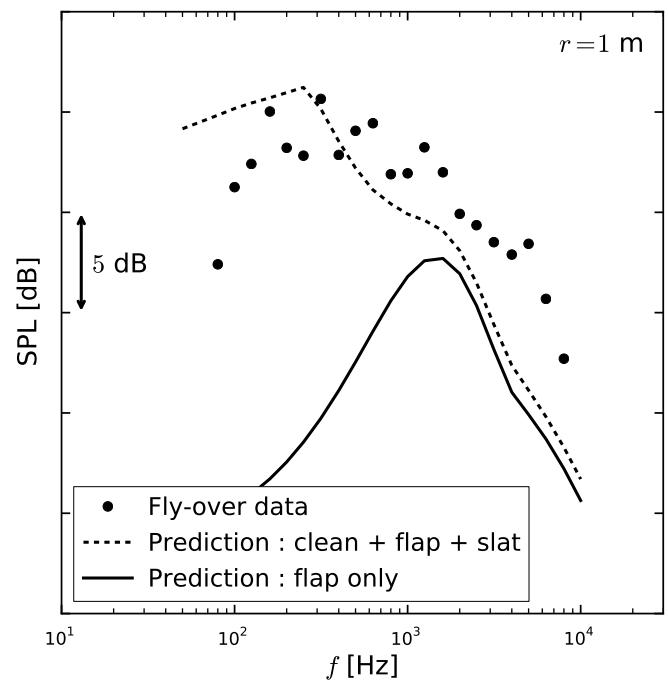

(b) $\delta_{f}=40^{\circ}, U_{0}=70.6 \mathrm{~m} / \mathrm{s}$

Figure 8: Comparison of the A319 fly-over noise levels (symbols) and the corresponding full-scale prediction (lines) for two flap deflection angles $\left(\delta_{f}=20^{\circ}, 40^{\circ}\right) . \phi=0^{\circ}, \theta=-90^{\circ}$.

single flap profile geometry and, therefore, is not expected to be representative for all types of aircraft.

\section{Conclusion}

An empirical prediction model for flap tip noise was presented which is based on acoustic and flow measurements at an isolated flap with a flat tip (modified A320 geometry). The database used to formulate the prediction scheme was obtained through dedicated experimental measurements in the Acoustic Wind Tunnel Braunschweig between 2008 and 2011. Details about the experiments and the data analysis can be found in Rossignol. ${ }^{13}$

These measurements enabled the quantification of flap tip noise as a function of free-stream velocity and flap deflection angle. Use of the cross-flow velocity, defined in Rossignol ${ }^{13}$ as the flow velocity normal 
to the tip, in the spanwise direction, was found to be an important parameter characterizing the noise production mechanism. Using this parameter to normalize the data, renders sound pressure levels which are independent of the free-stream velocity and flap deflection angle. Therefore, a single representation of the flap tip noise spectral shape, can be obtained by a least-squares fit of the normalized data. In a similar fashion, a functional representation of the noise directivity was obtained.

The prediction scheme described herein was implemented in a complete airframe noise prediction program $^{18}$ and compared against flyover measurements of a Boeing B747-400 and an Airbus A319. It was found that flap tip noise plays an important role only at high frequencies when considering the complete aircraft high-lift noise. Knowledge of the flap tip noise peak frequency and its high frequency spectral decay could therefore be sufficient to correctly predict its contribution to the overall highlift noise levels. The fly-over noise levels at high frequencies remain, however, slightly underpredicted suggesting that other noise sources (flap tracks, installation effects) might also be of importance at these frequencies. This issue could not be resolved here.

For the prediction of the full scale fly-over noise levels, an effective flap angle $20^{\circ}$ higher than the actual geometrical angle had to be considered to compute the cross-flow velocities. This assumption is justified by different flow condition in real flight compared to the wind tunnel experiment. It remains unclear how the geometrical flap deflection angle in the wind tunnel experiment can be properly extrapolated to full scale (or to other model cases). This needs to be tackled in future research efforts. The prediction model in its actual version does not account for tip shape modifications. To investigate this aspect, more measurement data at different model geometries are required.

\section{Acknowledgments}

The author gratefully acknowledges Lufthansa German Airlines for providing the Boeing B747-400 and Airbus A319 fly-over noise measurements data.

\section{Appendix A : Spectral shape model coefficients}

\begin{tabular}{cr|cr} 
Coefficient & \multicolumn{1}{c}{ Low $\mathcal{S} t_{c}$} & Coefficient & \multicolumn{1}{c}{ High $\mathcal{S} t_{c}$} \\
\hline$l_{0}$ & $8.2489 \times 10^{+1}$ & $h_{0}$ & $9.0495 \times 10^{+1}$ \\
$l_{1}$ & $4.6852 \times 10^{+0}$ & $h_{1}$ & $-2.1459 \times 10^{-1}$ \\
$l_{2}$ & $-5.1103 \times 10^{-1}$ & $h_{2}$ & $8.3252 \times 10^{-4}$ \\
$l_{3}$ & $2.3800 \times 10^{-2}$ & $h_{3}$ & $-1.3673 \times 10^{-6}$ \\
$l_{4}$ & $-5.5749 \times 10^{-4}$ & & \\
$l_{5}$ & $6.4265 \times 10^{-6}$ & & \\
$l_{6}$ & $-2.8910 \times 10^{-8}$ & & \\
\hline
\end{tabular}

Table 3: Regression coefficients of equations 3 and 4. 


\section{Appendix B : Directivity model coefficients}

\begin{tabular}{|c|c|c|c|c|}
\hline $\mathrm{f}[\mathrm{Hz}]$ & $m_{0}$ & $m_{1}$ & $m_{2}$ & $m_{3}$ \\
\hline 1000.0 & 0.00000114 & -0.00042264 & 0.03635690 & -0.67373313 \\
\hline 1250.0 & 0.00000139 & -0.00056227 & 0.06049393 & -1.50098107 \\
\hline 1600.0 & 0.00000032 & -0.00012172 & 0.01040979 & -0.06221714 \\
\hline 2000.0 & 0.00000006 & -0.00000240 & -0.00583854 & 0.27105025 \\
\hline 2500.0 & -0.00000019 & 0.00010166 & -0.01807377 & 0.52439897 \\
\hline 3150.0 & -0.00000035 & 0.00017228 & -0.02730976 & 0.73960937 \\
\hline 4000.0 & -0.00000029 & 0.00017178 & -0.03251828 & 0.90282649 \\
\hline 5000.0 & -0.00000023 & 0.00013543 & -0.02669603 & 0.88094234 \\
\hline 6300.0 & -0.00000048 & 0.00022256 & -0.03222015 & 1.00861554 \\
\hline 8000.0 & -0.00000089 & 0.00038304 & -0.04818164 & 1.38732002 \\
\hline 10000.0 & -0.00000131 & 0.00056889 & -0.07198845 & 2.03465714 \\
\hline 12500.0 & -0.00000171 & 0.00074516 & -0.09315186 & 2.48163075 \\
\hline 16000.0 & -0.00000138 & 0.00062285 & -0.08491453 & 2.71737498 \\
\hline $\mathrm{f}[\mathrm{Hz}]$ & $k_{0}$ & $k_{1}$ & $k_{2}$ & $k_{3}$ \\
\hline 1000.0 & 0.00000114 & -0.00042264 & 0.03635690 & -0.67373313 \\
\hline 1250.0 & 0.00000139 & -0.00056227 & 0.06049393 & -1.50098107 \\
\hline 1600.0 & 0.00000032 & -0.00012172 & 0.01040979 & -0.06221714 \\
\hline 2000.0 & 0.00000006 & -0.00000240 & -0.00583854 & 0.27105025 \\
\hline 2500.0 & -0.00000019 & 0.00010166 & -0.01807377 & 0.52439897 \\
\hline 3150.0 & -0.00000035 & 0.00017228 & -0.02730976 & 0.73960937 \\
\hline 4000.0 & -0.00000029 & 0.00017178 & -0.03251828 & 0.90282649 \\
\hline 5000.0 & -0.00000023 & 0.00013543 & -0.02669603 & 0.88094234 \\
\hline 6300.0 & -0.00000048 & 0.00022256 & -0.03222015 & 1.00861554 \\
\hline 8000.0 & -0.00000089 & 0.00038304 & -0.04818164 & 1.38732002 \\
\hline 10000.0 & -0.00000131 & 0.00056889 & -0.07198845 & 2.03465714 \\
\hline 12500.0 & -0.00000171 & 0.00074516 & -0.09315186 & 2.48163075 \\
\hline 16000.0 & -0.00000138 & 0.00062285 & -0.08491453 & 2.71737498 \\
\hline
\end{tabular}

Table 4: Regression coefficients of equation 5. 


\section{References}

${ }^{1}$ Howe, M. S., "On the generation of side-edge flap noise," Journal of Sound and Vibration, Vol. 80, No. 4, Feb. 1982, pp. $555-573$

${ }^{2}$ Ahtye, W. F., R. Wendell, M., and William C., M., "Wing and flap noise measured by near- and far-field crosscorrelation techniques," AIAA 5th Aeroacoustics Conference, March 1979.

${ }^{3}$ Brooks, T. and Marcolini, M., "Airfoil Tip Vortex Formation Noise," AIAA Journal, Vol. 24, No. 2, 1986, pp. 246252.

${ }^{4}$ Brooks, T. and Humphreys Jr., W. M., "Flap edge aeroacoustic measurements and prediction," 6th AIAA/CEAS Aeroacoustics Conference, 2000.

${ }^{5}$ Guo, Y., Joshi, M., Bent, P., and Yamamoto, K., "Surface pressure fluctuations on aircraft flaps and their correlation with far-field noise," Journal of fluid mechanics, Vol. 415, 2000, pp. 175-202.

${ }^{6}$ Guo, Y. and Yamamoto, K., "Component-Based Empirical Model for High-Lift System Noise Prediction," Journal of Aircraft, Vol. 40, No. 5, 2003, pp. 914-922.

${ }^{7}$ Koop, L., Aktive und passive Strömungsbeeinflussung zur Reduzierung der Schallabstrahlung an Hinterkantenklappen von Tragflügeln., Ph.D. thesis, Technische Universität Berlin, 2005.

${ }^{8}$ Sen, R., Hardy, B., and Miller, G., "Airframe noise sub-component definition and model," Tech. Rep. CR-2004-213255, NASA, 2004.

${ }^{9}$ Guo, Y., "Prediction of flap side edge noise," AIAA Journal, Vol. 1, 1999, pp. 35-45.

${ }^{10}$ Guo, Y., "Modeling of noise reduction by flap side edge fences," AIAA Journal, , No. AIAA-00-2065, 2000.

${ }^{11}$ Hardin, J. C., "Noise generation at the side edges of flaps," AIAA Journal, Vol. 18, No. 5, 1980, pp. 549-552.

${ }^{12}$ Sen, R., "Local dynamics and acoustics in a simple 2D model of airfoil lateral-edge noise," 2nd AIAA/CEAS Aeroacoustics Conference, 1996.

${ }^{13}$ Rossignol, K.-S., "Development of an empirical prediction model for flap side-edge noise." Proceedings of the 16th AIAA/CEAS Aeroacoustics Conference, Stockholm, Sweden, June 2010, No. AIAA 2010-3836, 2010.

${ }^{14}$ Mueller, T. J., editor, Aeroacoustic Measurements, No. ISBN : 978-3-540-41757-6, Springer, 2002.

${ }^{15}$ Brooks, T. F. and Humphreys, W. M., "Effect of directional array size on the measurement of airframe noise components," 5th AIAA/CEAS Aeroacoustics Conference, No. 99-1958, May 1999.

${ }^{16}$ Amiet, R., "Refraction of sound by a shear layer," Journal of Sound and Vibration, Vol. 58, No. 4, June 1978, pp. $467-482$.

${ }^{17}$ Hayes, J., Horne, W., Soderman, P., and Bent, P., "Airframe noise characteristics of a 4.7AIAA/CEAS Aeroacoustics Conference", No. AIAA/CEAS-97-1594, 1997.

${ }^{18}$ Dobrzynski, W., Rossignol, K.-S., and Almoneit, D., "Handbuch zum modularen Quellmodell für das Umströmungsgeräusch von Verkehrsflugzeugen PROFAN (PRediction MOdel For Airframe Noise), Version 3.2." Tech. rep., DLR, February 2011. 